Proceedings of the 43rd "Jaszowiec", International School and Conference on the Physics of Semiconductors, Wisła 2014

\title{
Mathematical Structure of Bosonic and Fermionic Jack States and Their Application in Fractional Quantum Hall Effect
}

\author{
B. Kuśmierz ${ }^{a, *}$, Y.-H. Wu ${ }^{b}$ AND A. WÓJs ${ }^{a}$ \\ ${ }^{a}$ Institute of Physics, Wrocław University of Technology, Wybrzeże Wyspiańskiego 27, 50-370 Wrocław, Poland \\ ${ }^{b}$ Department of Physics, Pennsylvania State University, University Park PA, 16802, USA
}

\begin{abstract}
Fractional quantum Hall effect is a remarkable behaviour of correlated electrons, observed exclusively in two dimensions, at low temperatures, and in strong magnetic fields. The most prominent fractional quantum Hall state occurs at Landau level filling factor $\nu=1 / 3$ and it is described by the famous Laughlin wave function, which (apart from the trivial Gaussian factor) is an example of Jack polynomial. Fermionic Jack polynomials also describe another pair of candidate fractional quantum Hall states: Moore-Read and Read-Rezayi states, believed to form at the $\nu=1 / 2$ and $3 / 5$ fillings of the second Landau level, respectively. Bosonic Jacks on the other hand are candidates for certain correlated states of cold atoms. We examine here a continuous family of fermionic Jack polynomials whose special case is the Laughlin state as approximate wave functions for the $1 / 3$ fractional quantum Hall effect.
\end{abstract}

DOI: 10.12693/APhysPolA.126.1134

PACS: 71.10.Pm, 73.43.Cd

\section{Introduction}

Numerical calculations of wave functions for fractional quantum Hall (FQH) effect [1] involve exact diagonalization of the electron-electron interaction Hamiltonian [2]. The computational power needed for these calculations grows exponentially with the number of particles. One of several methods proposed to decrease computation time involves the expansion of certain Jack polynomials, called "Jacks" [3] (the antisymmetric/fermionic Jacks for the electrons, and the standard symmetric Jacks for the analogous states of bosonic cold atoms), indexed by a negative rational number in the monomial basis. Specifically, the coefficients of the Jack polynomials in the monomial basis are the unnormalized expansion coefficients of the corresponding quantum many-electron Jack states in the configuration basis.

The FQH wave functions which have been realised to be Jack states include Laughlin (at the Landau level filling factor $\nu=1 / r$, with $r$ odd) [4], Moore-Read $(\nu=1 / 2)$ [5] and Read-Rezayi $(\nu=3 / 5)$ [6] states. Most computations are carried out in spherical geometry introduced by Haldane [7]. The Jack polynomials may be mapped onto the Haldane sphere and then compared with the exact numerical ground states from Hamiltonian diagonalization by overlapping both functions.

\section{Jack polynomials}

The Jack polynomial is a symmetric polynomial indexed by two parameters: partition $\lambda$ and a real number $\alpha$ [8]. The partition is a sequence $\lambda=\left(\lambda_{1}, \lambda_{2}, \lambda_{3} \ldots\right)$,

${ }^{*}$ corresponding author of non-negative integers in weakly decreasing order. The non-zero elements of the sequence are parts of $\lambda$. The number of parts is the length of $\lambda$ and it is denoted by $\ell(\lambda)$. The sum of parts of $\lambda$ is called weight and it is denoted by $|\lambda|$. The symbol $m(\lambda, i)$ is the number of parts of $\lambda$ equal to $i$. One can define natural order as follows: $\lambda$ dominates $\mu$ (written as: $\lambda \geq \mu$ ) when for every natural number $i$, the sum of the first $i$ parts of $\lambda$ is greater or equal to the sum of first $i$ parts of $\mu . \Lambda_{n}$ is the ring of symmetric polynomials of $n$ variables, defined as a direct sum over $k$ of sets of homogeneous, symmetric polynomials of $n$ variables and degree $k$, including also the zero polynomial. The monomial symmetric functions $m_{\lambda}$ are defined as:

$$
m_{\lambda}=\sum_{\alpha \in A} x_{1}^{\alpha_{1}} \cdot x_{2}^{\alpha_{2}} \cdot \ldots \cdot x_{n}^{\alpha_{n}},
$$

where $A$ is the set of all distinct permutations of $\lambda$.

The Jack polynomials are defined as the eigenvectors of a certain differential operator: Hamiltonian in the Calogero-Sutherland-Moser model given by

$$
H_{\mathrm{CMS}}=\alpha \sum_{i}\left(x_{i} \partial_{i}\right)^{2}+\sum_{i<j} \frac{x_{i}+x_{j}}{x_{i}-x_{j}}\left(x_{i} \partial_{i}-x_{j} \partial_{j}\right) \text {. }
$$

The Jack polynomials are well-defined for all positive and almost all negative values of parameter $\alpha$ (each Jack indexed by a particular partition $\lambda$ has a finite number of negative poles). The expansion of Jack in terms of monomial symmetric functions has non-zero elements only for those $m_{\mu}$ which are indexed by partitions $\mu$ dominated by the partition $\lambda$ of the Jack itself

$$
J_{\lambda}^{\alpha}=\sum_{\mu \leq \lambda} v_{\lambda \mu} m_{\mu} .
$$

The recursion formula for $v_{\lambda \mu}$ coefficients has been derived [9]. Starting from $v_{\lambda \lambda}=1$, other coefficients can be calculated using 


$$
v_{\lambda \mu}=\frac{1}{e_{\lambda}-e_{\theta}} \sum_{\mu \leq \theta \leq \lambda} C_{\mu \theta} v_{\lambda \mu} .
$$

For $e_{\lambda}$ - eigenvalue of $H_{\mathrm{CSM}}$ of $n$-variable Jack indexed by $\lambda$ :

$$
e_{\lambda}=\sum_{i=1}^{\ell(\lambda)}\left(\alpha \lambda_{i}^{2}+(n+1-2 i) \lambda_{i}\right)
$$

and $C_{\mu \theta}$ are non-zero coefficients only if there exist $i, j, l$ such: $\theta=\left(\mu_{1}, \mu_{2}, \ldots \mu_{i}-l, \ldots \mu_{j}+l, \ldots \mu_{n},\right) *$, for * - rearranging component of a vector, to a partition. Then, for $\mu_{i}-l \neq \mu_{j}+l$ :

$$
C_{\mu \theta}=\left(\mu_{i}-\mu_{j}\right) m\left(\theta, \mu_{i}-l\right) \cdot m\left(\theta, \mu_{j}+l\right),
$$

or half of this expression for $\mu_{i}-l=\mu_{j}+l$.

\section{Fermionic Jack polynomials}

Since the Jack polynomial is a symmetric function, it can only describe boson states. To obtain functions describing fermionic states one can use the canonical transformation from the ring of symmetric functions into the ring of antisymmetric functions - multiplication by Vandermonde determinant $\prod_{i<j}\left(x_{i}-x_{j}\right)$. The antisymmetrized Jack, also called the fermionic Jack [10] is hence defined as:

$$
S_{\lambda+\delta}^{\alpha}=J_{\lambda}^{\alpha} \prod_{i<j}\left(x_{i}-x_{j}\right),
$$

where $\delta=(n-1, n-2, \ldots 1,0)$, addition of partitions is defined by adding parts of partitions indexed by the same number.

Fermionic Jacks are the eigenvectors of the fermionic Laplace-Beltrami operator $-H_{\mathrm{LB}}^{\mathrm{F}}$ :

$$
H_{\mathrm{LB}}^{\mathrm{F}}=H_{\mathrm{KIN}}+\frac{1}{2}\left(\frac{1}{\alpha}-1\right) H_{\mathrm{INT}} .
$$

For

$$
H_{\mathrm{KIN}}=\sum_{i}\left(x_{i} \partial_{i}\right)^{2},
$$

and

$$
H_{\mathrm{INT}}=\sum_{i<j} \frac{x_{i}+x_{j}}{x_{i}-x_{j}}\left(x_{i} \partial_{i}-x_{j} \partial_{j}\right)-\frac{x_{i}^{2}+x_{j}^{2}}{\left(x_{i}-x_{j}\right)^{2}} .
$$

Using this property one can calculate the coefficients of fermionic Jacks in the Slater determinant basis [10, 11].

\section{Jack states}

Bosonic Laughlin wave function can be represented as a product of the Gaussian function and a symmetric Jack polynomial. Therefore an (unnormalized) bosonic state at filling factor $\nu=1 / r$ for even $r$ may be expressed as [12]:

$$
\Phi_{\mathrm{L}}^{r}\left(x_{1}, x_{2}, \ldots x_{n}\right) \exp \left(-\frac{1}{4 \ell_{\mathrm{B}}^{2}} \sum_{i}\left|x_{i}\right|^{2}\right) .
$$

For $\ell_{B}-$ magnetic length, where $\Phi_{\mathrm{L}}^{r}\left(x_{1}, x_{2}, \ldots x_{n}\right)$ is a Jack polynomial, given by

$$
\Phi_{\mathrm{L}}^{r}\left(x_{1}, x_{2}, \ldots x_{n}\right)=J_{\lambda^{0}(1, r)}^{\alpha_{1, r}}\left(x_{1}, x_{2}, \ldots x_{n}\right) .
$$

For $\lambda^{0}(1, r)$ given by $\lambda^{0}(1, r)=((n-1) r,(n-2) r, \ldots, r, 0)$ and $\alpha_{k, r}=-(k+1) /(r-1)$.

Fermionic Laughlin wave functions are also Jack states. The polynomial part of the $\nu=1 / r$ wave function for odd $r$ is a fermionic Jack polynomial indexed by partition $\lambda^{0}(1, r)$ and $\alpha=-2 /(r-2)$.

\section{Jack polynomials and pseudopotential}

The Laughlin wave function describes fractional quantum Hall effect states under assumption of special form of the $e-e$ interaction pseudopotential (interaction energy $V$ as a function of relative pair angular momentum $m)$ - short-range repulsion. This pseudopotential takes non-zero value only for $V(m=1)$. We examined the overlaps of fermionic Jack functions indexed by partition $\lambda^{0}(1, r)$ and the FQH wave functions obtained from diagonalization of a Hamiltonian with the special form of the interaction pseudopotential. This peudopotential is nonzero only at $m=1$ and 3 , and may be indexed by a single real number $\beta$ defined as follows:

$V(m=1)=1, V(m=3)=\beta$.

We examine the overlaps in the neighbourhood of $\alpha_{k, r}$ and for $\beta=0$ we expect to find the overlap equal to 1 for this point (Fig. 1).

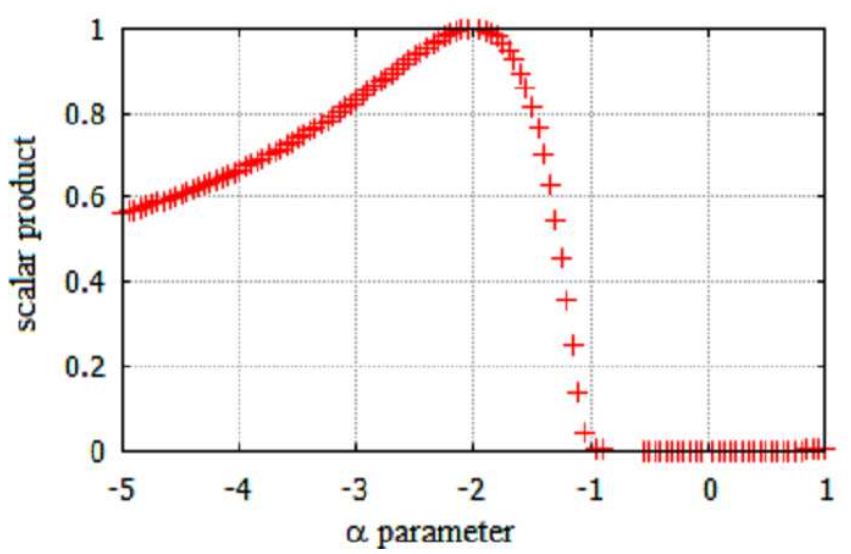

Fig. 1. Overlap of the 7 particle wave function for state $\nu=1 / 3$, modeled with short-range repulsionpseudopotential $(\beta=0)$ and the fermionic Jack indexed by the partition $\lambda^{*}=(18,15,12,9,6,3,0)$ in the neighborhood of $\alpha=-2$.

Along with the increase of $\beta$, the overlaps of functions slightly decreased, but for $\beta$ less than roughly 0.5 the shape of the curve remains almost the same (Fig. 2). When $\beta$ drops to about 0.5 (i.e., the shape of the pseudopotential through the leading three values at $m=1$, 3,5 is no longer strictly convex [13]) the fermionic Jack rapidly loses overlap with the exact ground state wave function and it is no longer a good approximation of the system (Fig. 3). 


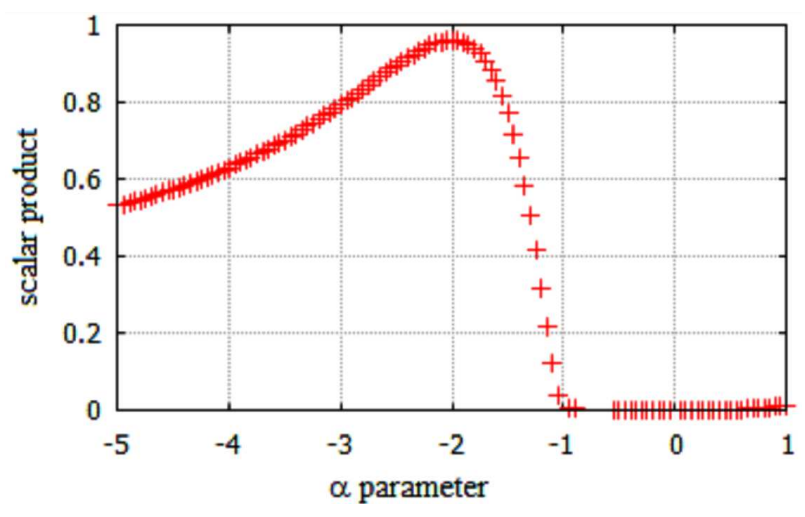

Fig. 2. Overlap of the 7 particle wave function for state $\nu=1 / 3$, modeled with generalized pseudopotential for $\beta=0.3$ and the fermionic Jack indexed by a partition $\lambda^{*}$ in the neighborhood of -2 .

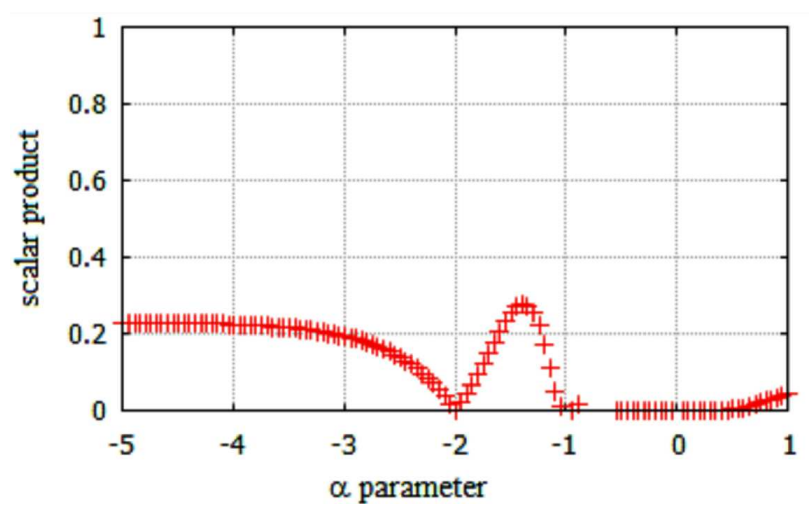

Fig. 3. Overlap of the 7 particle wave function for state $\nu=1 / 3$, modeled with generalized pseudopotential for $\beta=0.6$ and the fermionic Jack indexed by a partition $\lambda^{*}$ in the neighborhood of -2 .

\section{Conclusions}

We examined fermionic Jack polynomials as the candidates for approximate FQH wave functions for the special type of $e-e$ interaction pseudopotential relevant for the fermionic Laughlin state $\nu=1 / 3$ in the lowest Landau level. We have performed the calculations for fermionic Jacks indexed by a particular partition which produces exact description of the Laughlin wave function for a particular choice of parameter $\alpha=-2$, and for exact manyelectron ground states of a model interaction pseudopotential with repulsion range controlled by the parameter $\beta$. The expansion coefficients of fermionic Jack polynomial in the Slater determinant basis are continuous with respect to parameter $\alpha$. Moreover, the Hamiltonian as a matrix is a continuous function of its coefficients. Therefore obtained overlaps are close to the unity for small $\beta$ and in close neighbourhood of $\alpha=-2$. However, when the range of repulsion is increased to the point when pseudopotential in no longer convex, the resulting ground state is no longer described by the Jack polynomial.

\section{Acknowledgments}

We acknowledge support from the Polish NCN Grant No. 2011/01/B/ST3/04504 and use of computing facilities of the Cyfronet and WCSS, both parts of PL-Grid Infrastructure.

\section{References}

[1] D.C. Tsui, H.L. Störmer, A.C. Gossard, Phys. Rev. Lett. 48, 1559 (1982); J.K. Jain, Composite Fermions, Cambridge University Press, Cambridge 2007.

[2] J.J. Quinn, A. Wójs, J. Phys. Condens. Matter 12, R265 (2000); J.J. Quinn, A. Wójs, K.-S. Yi, G. Simion, Phys. Rep. 481, 29 (2009).

[3] H. Jack, Proc. R. Soc. Edin. Sect. A 69, 1 (1970/1971).

[4] R. Laughlin, Phys. Rev. Lett. 50, 1395 (1983).

[5] G. Moore, N. Read, Nucl. Phys. B 360, 362 (1991).

[6] N. Read, E.H. Rezayi, Phys. Rev. B 59, 8084 (1999).

[7] F.D.M. Haldane, Phys. Rev. Lett. 51, 605 (1983).

[8] R. Stanley, Adv. Math. 77, 76 (1988).

[9] K. Sogo, J. Math. Phys. 35, 2282 (1994); L. Lapointe, A. Lascoux, J. Morse, Elec. J. Combin. N1, 7 (2000); L. Lapointe, A. Lascoux, J. Morse, Int. Math. Res. Notices 18, 957 (1998).

[10] B.A. Bernevig, N. Regnault, Phys. Rev. Lett. 103, 206801 (2009).

[11] R. Thomale, B. Estienne, N. Regnault, B.A. Bernevig, Phys. Rev. B 84, 045127 (2011).

[12] B.A. Bernevig, F.D.M. Haldane, Phys. Rev. Lett. 100, 246802 (2008); Phys. Rev. B 77, 184502 (2008); W. Baratta, P.J. Forrester, Nucl. Phys. B 843, 362 (2011).

[13] J.J. Quinn, A. Wójs, Physica E 6, 1 (2000); A. Wójs, J.J. Quinn, Solid State Commun. 108, 493 (1998). 